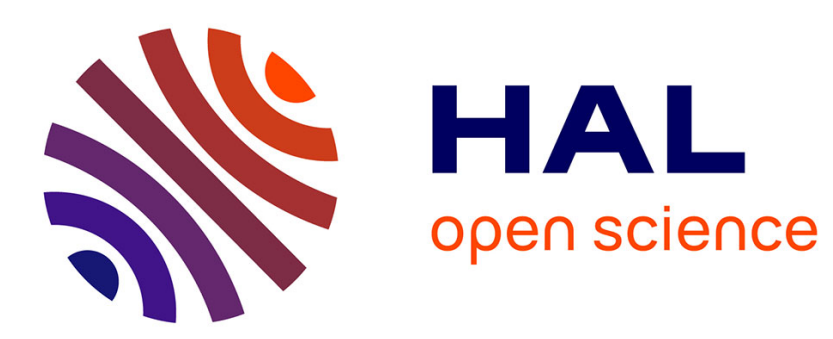

\title{
On simple scheme of finite/fixed-time control design
}

Konstantin Zimenko, Andrey Polyakov, Denis Efimov, Wilfrid Perruquetti

\section{To cite this version:}

Konstantin Zimenko, Andrey Polyakov, Denis Efimov, Wilfrid Perruquetti. On simple scheme of finite/fixed-time control design. International Journal of Control, 2020, 93 (6), 10.1080/00207179.2018.1506889 . hal-01872533

\section{HAL Id: hal-01872533 \\ https://hal.inria.fr/hal-01872533}

Submitted on 12 Sep 2018

HAL is a multi-disciplinary open access archive for the deposit and dissemination of scientific research documents, whether they are published or not. The documents may come from teaching and research institutions in France or abroad, or from public or private research centers.
L'archive ouverte pluridisciplinaire HAL, est destinée au dépôt et à la diffusion de documents scientifiques de niveau recherche, publiés ou non, émanant des établissements d'enseignement et de recherche français ou étrangers, des laboratoires publics ou privés. 


\title{
On simple scheme of finite/fixed-time control design*
}

\author{
K. Zimenko ${ }^{\mathrm{a}}$, A. Polyakov ${ }^{\mathrm{a}, \mathrm{b}, \mathrm{c}}$, D. Efimov ${ }^{\mathrm{a}, \mathrm{b}, \mathrm{c}}$ and W. Perruquetti ${ }^{\mathrm{b}, \mathrm{c}}$ \\ ${ }^{a}$ Department of Control Systems and Informatics, ITMO University, 49 Kronverkskiy av.,

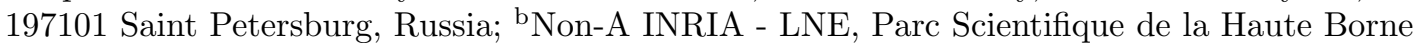 \\ 40, avenue Halley Bat.A, Park Plaza 59650 Villeneuve d'Ascq, France; ${ }^{\circ}$ CRIStAL \\ (UMR-CNRS 9189), Ecole Centrale de Lille, BP 48, Cite Scientifique, 59651 \\ Villeneuve-d'Ascq, France
}

\begin{abstract}
Control laws are designed for stabilization of a chain of integrators of arbitrary degree in finite and fixed time. Presented control laws are obtained with use of Lyapunov function method and homogeneity concept. The present analysis is based on use of explicitly defined Lyapunov function that is the hallmark with respect to similar works. This analysis allows to get simple procedure of parameters tuning and obtain new estimates for settling-time function. The theoretical results are supported by numerical examples.
\end{abstract}

\section{KEYWORDS}

Finite-time stabilization; fixed-time stabilization; homogeneity

\section{Introduction}

Motivated by modern control applications, the finite-time and fixed-time stabilization feedback design is continuing to be the subject of numerous studies (see, for example, Bacciotti \& Rosier, 2005; Bhat \& Bernstein, 2000; Cruz-Zavala \& Moreno, 2017; Orlov, 2004; Polyakov, Efimov, \& Perruquetti, 2015, etc.). Among these studies and many others, particular attention is paid to finite/fixed-time stabilization of integrator chains as a basic model for demonstration and further extension for multi-input multi-output linear plants. Interest to the disturbed integrator chain control systems is additionally motivated by different mechanical and electromechanical applications (see, for example, Chernousko, Ananevskii, \& Reshmin, 2008; Utkin, Guldner, \& Shi, 2009). However, most of existing control laws are discontinuous (as, for example, in Cruz-Zavala \& Moreno, 2017; Harmouche, Laghrouche, Chitour, \& Hamerlain, 2016), designed to stabilize the chain of integrators only of a certain order (for second order Orlov, Aoustin, \& Chevallereau, 2011; Sua \& Zheng, 2015; for third order Sanchez \& Moreno, 2013; Trivedi \& Bandyopadhyay, 2011, etc.), or even depending directly on the initial conditions (Sanchez \& Moreno, 2013).

\footnotetext{
*A preliminary version of this work (Zimenko, Polyakov, Efimov, \& Perruquetti, in press) does not contain proofs and the case of continuous fixed-time control design.

CONTACT K. Zimenko. Email: kostyazimenko@gmail.com
} 
The present paper is devoted to Lyapunov-based controller design which stabilizes the arbitrary order integrator chain in finite/fixed time. Presented control algorithms are obtained with use of Lyapunov function method and homogeneity concept.

The paper (Polyakov et al., 2015) is related to development of robust finite-time and fixed-time stabilization controllers, which depend on implicitly defined homogeneous Lyapunov function. For practical implementation of these control algorithms it is required to use special computational procedures for calculating Implicit Lyapunov Function (ILF). In (Zimenko, Polyakov, \& Efimov, 2016) the finite-time control law has been modified to explicit form, where instead of ILF the homogeneous norm is used. Then the difference between ILF and homogeneous norm is considered as additional external disturbance, that has led to a more complex analysis and parameters tuning procedure. In comparison with these works the novelty of the present paper is as follows:

- new finite-time and fixed-time control algorithms are proposed in explicit form for the system of arbitrary order;

- stability analysis is based on the use of a Lyapunov function proposed in explicit form;

- simple procedures of parameters tuning are established;

- new estimates for settling-time function are presented.

It is also shown that fixed-time convergence can be achieved by changing the homogeneity degree in hybrid control algorithm.

In addition, the proposed controllers homogenize the system that implies robustness abilities to external perturbations and time-delays (see, for example, Bernuau, Polyakov, Efimov, \& Perruquetti, 2013; Efimov, Polyakov, Perruquetti, \& Richard, 2016; Zimenko, Efimov, Polyakov, \& Perruquetti, 2017).

The paper is organized in the following way. The problem formulation is presented in Section 2. Section 3 recalls some basics on finite/fixed-time stability and weighted homogeneity. Section 4 presents the main result on finite-time and fixed-time controllers design. Simulation results are shown in Section 5 for performance illustration of proposed control algorithms. Finally, concluding remarks are given in Section 6 .

Through the paper the following notation will be used: $\mathbb{R}$ is the set of real numbers; $\mathbb{R}_{+}=\{x \in \mathbb{R}: x>0\} ; \operatorname{diag}\left\{\lambda_{i}\right\}_{i=1}^{n}$ is a diagonal matrix with elements $\lambda_{i}$; for $P \in \mathbb{R}^{n \times n}$ the relation $P>0(P<0, P \geq 0, P \leq 0)$ means that the symmetric matrix $P=P^{T}$ is positive (negative) definite (semidefinite); a series of integers $1,2, \ldots, n$ is denoted by $\overline{1, n}$.

\section{Problem formulation}

Consider a chain of $n$ integrators

$$
\dot{x}=A x+b u,
$$


where $x \in \mathbb{R}^{n}$ is the state vector, $u \in \mathbb{R}$,

$$
A=\left(\begin{array}{ccccc}
0 & 1 & 0 & \cdots & 0 \\
0 & 0 & 1 & \cdots & 0 \\
\vdots & \vdots & \vdots & \ddots & \vdots \\
0 & 0 & 0 & \cdots & 1 \\
0 & 0 & 0 & \cdots & 0
\end{array}\right) \text { and } b=\left(\begin{array}{c}
0 \\
0 \\
\vdots \\
0 \\
1
\end{array}\right)
$$

The objective of the present paper is to design Lyapunov-based controllers, which stabilize the arbitrary order system (1) to the origin in finite/fixed time.

\section{Preliminaries}

\subsection{Finite-time and fixed-time stability}

Let us consider the system

$$
\dot{x}=f(t, x), \quad x(0)=x_{0},
$$

where $x \in \mathbb{R}^{n}$ is the state vector, $f \in \mathbb{R}_{+} \times \mathbb{R}^{n} \rightarrow \mathbb{R}^{n}$ is a vector field. If the vector field $f$ is discontinuous with respect to $x$, then the solutions of (2) fall into the area of differential inclusions and need to be understood in the sense of Filippov (1988). For any $x_{0} \in \mathbb{R}^{n}$ denote a corresponding solution by $\tilde{X}\left(t, x_{0}\right)$ for the instants $t \geq 0$ for which it exists, $\tilde{X}\left(0, x_{0}\right)=x_{0}$.

Definition 3.1 (Bhat \& Bernstein, 2000; Orlov, 2004). The origin of (2) is said to be globally finite-time stable if it is globally asymptotically stable and any solution $\tilde{X}\left(t, x_{0}\right)$ of $(2)$ reaches the equilibrium point at some finite time moment, i.e., $\tilde{X}\left(t, x_{0}\right)=0, \forall t \geq T\left(x_{0}\right)$, where $T: \mathbb{R}^{n} \rightarrow \mathbb{R}_{+} \cup\{0\}$ is the settling-time function.

Definition 3.2 (Polyakov, 2012). The origin of (2) is said to be fixed-time stable if it is globally finite-time stable and the settling-time function $T\left(x_{0}\right)$ is bounded, i.e., $\exists T_{\max }>0: T\left(x_{0}\right) \leq T_{\max }, \forall x_{0} \in \mathbb{R}^{n}$.

Definition 3.3 (Polyakov, 2012). The set $M$ is said to be globally finite-time attractive for $(2)$ if any solution $\tilde{X}\left(t, x_{0}\right)$ of $(2)$ reaches $M$ in some finite time moment $t=T\left(x_{0}\right)$ and remains there $\forall t \geq T\left(x_{0}\right), T: \mathbb{R}^{n} \rightarrow \mathbb{R}_{+} \cup\{0\}$ is the settling-time function.

Definition 3.4 (Polyakov, 2012). The set $M$ is said to be fixed-time attractive for (2) if it is globally finite-time attractive and the settling-time function $T\left(x_{0}\right)$ is globally bounded by some number $T_{\max }>0$.

Theorem 3.5 (Bhat \& Bernstein, 2000; Harmouche et al., 2016). Suppose there exists a positive definite $C^{1}$ function $V$ defined on an open neighborhood of the origin $D \subset \mathbb{R}^{n}$ and real numbers $C>0$ and $\sigma \geq 0$, such that the following condition is true for the system (2)

$$
\dot{V}(x) \leq-C V^{\sigma}(x), \quad x(t) \in D \backslash\{0\} .
$$

Then depending on the value $\sigma$ the origin is stable with different types of convergence: 
- if $\sigma=1$, the origin is exponentially stable;

- if $0 \leq \sigma<1$, the origin is finite-time stable and

$$
T\left(x_{0}\right) \leq \frac{1}{C(1-\sigma)} V_{0}^{1-\sigma},
$$

where $V_{0}=V\left(x_{0}\right)$;

- if $\sigma>1$ the origin is asymptotically stable and, for every $\epsilon$, the set $B(0, \epsilon)=$ $\{x \in D: V(x)<\varepsilon\}$ is fixed-time attractive with

$$
T_{\max }=\frac{1}{C(\sigma-1) \varepsilon^{\sigma-1}} .
$$

If $D=\mathbb{R}^{n}$ and function $V$ is radially unbounded, then the system (2) is globally stable with respect to the equilibrium point.

\subsection{Weighted homogeneity}

For $r_{i} \in \mathbb{R}_{+}, i=\overline{1, n}, \rho>2 \max _{i} r_{i}$ and $\lambda>0$ define vector of weights $r=$ $\left(r_{1}, \ldots, r_{n}\right)^{T}$, dilation matrix $D_{r}(\lambda)=\operatorname{diag}\left\{\lambda^{r_{i}}\right\}_{i=1}^{n}$ and homogeneous norm

$$
\|x\|_{r}=\left(\sum_{i=1}^{n}\left|x_{i}\right|^{\frac{\rho}{r_{i}}}\right)^{\frac{1}{\rho}} .
$$

Note that under introduced restriction on $\rho$ the homogeneous norm is a continuously differentiable function out of the origin.

Definition 3.6 (Zubov, 1958). A function $g: \mathbb{R}^{n} \rightarrow \mathbb{R}$ (vector field $f: \mathbb{R}^{n} \rightarrow \mathbb{R}^{n}$ ) is said to be $r$-homogeneous of degree $d$ if $g\left(D_{r}(\lambda) x\right)=\lambda^{d} g(x)\left(f\left(D_{r}(\lambda) x\right)=\right.$ $\left.\lambda^{d} D_{r}(\lambda) f(x)\right)$ for fixed $r$, all $\lambda>0$ and $x \in \mathbb{R}^{n}$.

Obviously, homogeneous norm is $r$-homogeneous of degree 1. Introduce the following compact set (homogeneous sphere) $\mathbb{S}_{r}=\left\{x \in \mathbb{R}^{n}:\|x\|_{r}=1\right\}$, then for any $x \in \mathbb{R}^{n}$ there is $y \in \mathbb{S}_{r}$ such that $x=D_{r}(\lambda) y$ for $\lambda=\|x\|_{r}$.

Theorem 3.7 (Bacciotti \& Rosier, 2005). Let $f: \mathbb{R}^{n} \rightarrow \mathbb{R}^{n}$ be defined on $\mathbb{R}^{n}$ and be a continuous $r$-homogeneous vector field with degree $d(d<0)$. If the origin of the system

$$
\dot{x}=f(x)
$$

is locally asymptotically stable then it is globally asymptotically stable (globally finitetime stable) and there exists a continuously differentiable Lyapunov function $V$ which is $r$-homogeneous of degree $v>-d$.

By definition of homogeneity there exist constants $c_{1}, c_{2}>0$ such that

$$
c_{1}\|x\|_{r}^{v} \leq V(x) \leq c_{2}\|x\|_{r}^{v} .
$$

The $r$-homogeneity presented in Definition 3.6 is introduced for some $r$ and all 
$\lambda>0$. Restricting the set of admissible values for $\lambda$ the local homogeneity concept has been introduced in (Andrieu, Praly, \& Astolfi, 2008; Bernuau et al., 2013; Zubov, 1958).

\section{Main result}

For $\mu \in(0,1]$ and the vector of weights $r=(l+(n-1) \mu, l+(n-2) \mu, \cdots, l)^{T}$, $r_{\max }=\max _{1 \leq j \leq n} r_{j}=l+(n-1) \mu, r_{\min }=\min _{1 \leq j \leq n} r_{j}=l$ denote the dilation matrix $D_{r}(\lambda)=\operatorname{diag}\left\{\lambda^{r_{i}}\right\}_{i=1}^{n}$ and diagonal matrix $\tilde{H}=\operatorname{diag}\{-m+(n+1-i) \mu\}_{i=1}^{n}$, where $l \in \mathbb{R}_{+}: l \geq \mu, m \in \mathbb{R}_{+}: m>n \mu$.

Theorem 4.1. If the system of matrix inequalities:

$$
\begin{gathered}
X>0, \\
\alpha_{\max }(\tilde{H} X+X \tilde{H})+A X+X A^{T}+b y+y^{T} b^{T} \leq-\gamma X, \\
-\alpha_{\max }(\tilde{H} X+X \tilde{H})+A X+X A^{T}+b y+y^{T} b^{T} \leq-\gamma X,
\end{gathered}
$$

is feasible for some $X \in \mathbb{R}^{n \times n}, y \in \mathbb{R}^{1 \times n}, \gamma \in \mathbb{R}_{+}$and

$$
\alpha_{\max }=\left(1+\left(\sum\left|k_{i}\right|^{\frac{\rho}{\rho-r_{\min }}}\right)^{\frac{\rho-r_{\min }}{r_{\min }}}\right)^{\frac{r_{\min }}{\rho}}\left(\sum\left(\frac{1}{r_{i}}\right)^{\frac{\rho}{r_{\max }-r_{\min }}}\right)^{\frac{r_{\max }-r_{\min }}{\rho}}
$$

$P=X^{-1}, k=y X^{-1}$, then the control of the form

$$
u=\|x\|_{r}^{l-\mu} k D_{r}\left(\frac{1}{\|x\|_{r}}\right) x
$$

stabilizes the origin of the system (1) in a finite time and the settling-time function estimate has the form

$$
T\left(x_{0}\right) \leq \frac{2(m+l-\mu) V_{0}^{\mu}}{\mu \gamma c_{1}^{\mu}}
$$

where $V_{0}=\left(x_{0}^{T} H\left(\frac{1}{\left\|x_{0}\right\|_{r}}\right) P H\left(\frac{1}{\left\|x_{0}\right\|_{r}}\right) x_{0}\right)^{\frac{1}{2(m+l-\mu)}}, H(\lambda)=\operatorname{diag}\left\{\lambda^{-m+(n+1-i) \mu}\right\}_{i=1}^{n}$ and $c_{1}=\min _{\|x\|_{r}=1}\left\{\left(x^{T} P x\right)^{\frac{1}{2(m+l-\mu)}}\right\}$.

Proof. Consider the Lyapunov candidate function in the next form:

$$
V(x)=\left(x^{T} H\left(\frac{1}{\|x\|_{r}}\right) P H\left(\frac{1}{\|x\|_{r}}\right) x\right)^{\frac{1}{2 m+2 l-2 \mu}} .
$$

The function $V$ is continuous, positive definite and radial unbounded on $\mathbb{R}^{n} \backslash\{0\}$. In addition, since $\lim _{x \rightarrow 0} V=0^{+}$the function $V$ can be continuously prolonged to the origin by setting $V(0)=0$. 
Note, that $V$ is homogeneous of degree 1 . The inequality (5) can be rewritten as

$$
c_{1}\|x\|_{r} \leq V \leq c_{2}\|x\|_{r}
$$

and $\|x\|_{r}^{-\mu} \geq c_{1}^{\mu} V^{-\mu}$. The parameter $c_{1}$ can be estimated on some grid, where $c_{1}=$ $\min _{\|x\|_{r}=1}\left\{\left(x^{T} P x\right)^{\frac{1}{2(m+l-\mu)}}\right\}$.

Define $\quad \alpha(x) \quad=\quad\left|\|x\|_{r}^{-1+\mu} \nabla_{x}\left(\|x\|_{r}\right) \dot{x}\right|$. Since $\quad \nabla_{x}\left(\|x\|_{r}\right) \dot{x}=$ $\frac{1}{\rho}\|x\|_{r}^{1-\rho} \sum \frac{\rho}{r_{i}}\left|x_{i}\right|^{\frac{\rho-r_{i}}{r_{i}}} \operatorname{sign}\left(x_{i}\right) \dot{x}_{i}=\|x\|_{r}^{1-\rho} \sum \frac{1}{r_{i}}\left|x_{i}\right|^{\frac{\rho-2 r_{i}}{r_{i}}} x_{i} \dot{x}_{i}$ it can be easily checked that $\alpha$ is homogeneous of degree $0,\|x\|_{r}$ is differentiable everywhere for $\rho>2 \max _{i} r_{i}$ and $0 \leq \alpha(x) \leq \alpha_{\max }$. Then the parameter $\alpha_{\max }$ can be found on the set $x \in \mathbb{S}_{r}$ using Hölder's inequality as follows:

$$
\begin{aligned}
& \left|\nabla_{x}\left(\|x\|_{r}\right) \dot{x}\right|=\left.\left.\frac{1}{\rho}\left|\sum \frac{\rho}{r_{i}}\right| x_{i}\right|^{\frac{\rho-r_{i}}{r_{i}}} \operatorname{sign}\left(x_{i}\right) \dot{x}_{i}\left|\leq \sum \frac{1}{r_{i}}\right| x_{i}\right|^{\frac{\rho-r_{i}}{r_{i}}}\left|\dot{x}_{i}\right| \leq \\
& \leq\left(\sum\left|x_{i}\right|^{\frac{\rho\left(\rho-r_{i}\right)}{\left(\rho-r_{\max }\right) r_{i}}}\right)^{\frac{\rho-r_{\max }}{\rho}}\left(\sum\left(\frac{1}{r_{i}}\left|\dot{x}_{i}\right|\right)^{\frac{\rho}{r_{\max }}}\right)^{\frac{r_{\max }}{\rho}} \leq \\
& \leq\left(\sum\left|\dot{x}_{i}\right|^{\frac{\rho}{r_{\min }}}\right)^{\frac{r_{\min }}{\rho}}\left(\sum\left(\frac{1}{r_{i}}\right)^{\frac{\rho}{r_{\max }-r_{\min }}}\right)^{\frac{r_{\max }-r_{\min }}{\rho}} \leq \\
& \leq\left(1+|u|^{\frac{\rho}{r_{\min }}}\right)^{\frac{r_{\min }}{\rho}}\left(\sum\left(\frac{1}{r_{i}}\right)^{\frac{\rho}{r_{\max }-r_{\min }}}\right)^{\frac{r_{\max }-r_{\min }}{\rho}} \leq \\
& \leq\left(1+\left(\sum\left|k_{i}\right|^{\frac{\rho}{\rho-r_{\min }}}\right)^{\frac{\rho-r_{\min }}{r_{\min }}}\right)^{\frac{r_{\min }}{\rho}}\left(\sum\left(\frac{1}{r_{i}}\right)^{\frac{\rho}{r_{\max }-r_{\min }}}\right)^{\frac{r_{\max }-r_{\min }}{\rho}}=\alpha_{\max } .
\end{aligned}
$$

Then by applying the inequalities (6)-(8) the time derivative of Lyapunov function $V$ along the trajectories of the system (1) can be estimated as follows

$$
\begin{aligned}
& \dot{V}=\frac{V^{1-2(m+l-\mu)}}{2(m+l-\mu)} 2 x^{T} H\left(\frac{1}{\|x\|_{r}}\right) P\left(\dot{H}\left(\frac{1}{\|x\|_{r}}\right) x+H\left(\frac{1}{\|x\|_{r}}\right) \dot{x}\right)= \\
& =\frac{V^{1-2(m+l-\mu)}}{2(m+l-\mu)} 2 x^{T} H\left(\frac{1}{\|x\|_{r}}\right) P\left(-\|x\|_{r}^{-1} \nabla_{x}\left(\|x\|_{r}\right) \dot{x} \tilde{H} H\left(\frac{1}{\|x\|_{r}}\right) x+\right. \\
& \left.\quad+\left(\|x\|_{r}\right)^{-\mu}\left(A H\left(\frac{1}{\|x\|_{r}}\right) x+b k H\left(\frac{1}{\|x\|_{r}}\right) x\right)\right)= \\
& =\frac{V^{1-2(m+l-\mu)}}{2(m+l-\mu)}\|x\|_{r}^{-\mu} 2 x^{T} H\left(\frac{1}{\|x\|_{r}}\right) P\left(-\|x\|_{r}^{-1+\mu} \nabla_{x}\left(\|x\|_{r}\right) \dot{x} \tilde{H}+A+b k\right) H\left(\frac{1}{\|x\|_{r}}\right) x \leq \\
& \leq-\frac{\gamma c_{1}^{\mu} V^{1+\mu-2(m+l)}}{2(m+l-\mu)} x^{T} H\left(\frac{1}{\|x\|_{r}}\right) P H\left(\frac{1}{\|x\|_{r}}\right) x=-\frac{\gamma c_{1}^{\mu}}{2(m+l-\mu)} V^{1-\mu}
\end{aligned}
$$

and

$$
T\left(x_{0}\right) \leq \frac{2(m+l-\mu) V_{0}^{\mu}}{\mu \gamma c_{1}^{\mu}},
$$

where $V_{0}=V\left(x_{0}\right)$.

Validity of Theorem 4.1 is thus concluded.

Remark 1. The finite-time control law (10) for $l=1$ coincides with the control presented in (Zimenko et al., 2016). In comparison with (Zimenko et al., 2016), presented analysis allows to relax obtained LMIs and simplify the procedure of parameters tuning. Also, new estimates for settling-time function are obtained. 
Remark 2. Since the inequalities (7), (8) are feasible at least for sufficiently small $\alpha$ (can be achieved by choosing sufficiently big $l \in \mathbb{R}_{+}$) the presented control scheme implies simple control parameters tuning: it requires to solve LMIs (6) and

$$
\begin{gathered}
\tilde{\alpha}(\tilde{H} X+X \tilde{H})+A X+X A^{T}+b y+y^{T} b^{T} \leq-\gamma X, \\
-\tilde{\alpha}(\tilde{H} X+X \tilde{H})+A X+X A^{T}+b y+y^{T} b^{T} \leq-\gamma X,
\end{gathered}
$$

for some $\tilde{\alpha} \in \mathbb{R}_{+}$and then choose such $l$ for which $\alpha_{\max } \leq \tilde{\alpha}$.

Remark 3. The parameter $m$ can be chosen large enough to have $\tilde{H} X+X \tilde{H} \leq 0$. In this case the inequality ( 7 ) can be ignored.

Remark 4. Parameters $l, m, \mu$ and $\gamma$ allows the upper bound of the settling time function (11) to be adjusted.

Remark 5. For $l=\mu \leq 1$ the control (10) is continuous outside the origin and globally bounded for all $x \in \mathbb{R}^{n}$ :

$$
\begin{aligned}
& u^{2}=x^{T} D_{r}\left(\frac{1}{\|x\|_{r}}\right) k^{T} k D_{r}\left(\frac{1}{\|x\|_{r}}\right) x \leq \varsigma\|x\|_{r}^{-2 m} x^{T} H\left(\frac{1}{\|x\|_{r}}\right) P H\left(\frac{1}{\|x\|_{r}}\right) x= \\
& =\varsigma\|x\|_{r}^{-2 m} V^{2 m} \leq \varsigma c_{2}^{2 m}
\end{aligned}
$$

where $\varsigma \in \mathbb{R}_{+}: k^{T} k \leq \varsigma P$.

For $\mu<\min \{l, 1\}$ the control (10) is continuous in the state variable $x$. If $\mu \rightarrow 0$ then the feedback (10) becomes a linear $u=k x$. If $\mu=l \leq 1$ the control is discontinuous at the origin and continuous outside. For practical realization of the control, the discontinuous feedback law can be replaced with a high-gain linear feedback if the system state is close to the origin as in (Polyakov et al., 2015).

Remark 6. It can be easily checked that the system (1), (10) is homogeneous of degree $-\mu$. Thus, the system has such qualitative stability properties of homogeneous systems as Input-to-State Stability with respect to additive perturbations in the righthand side of (1) and measurement noises in the feedback (see, for example, Bernuau et al., 2013).

Remark 7. Since the closed-loop system is $r$-homogeneous with negative degree, then in presence of any delay the system (1), (10) is globally asymptotically stable with respect to a compact set containing the origin independently of delay (see, for example, Zimenko et al., 2017).

Remark 8. The controller (10) can also be treated as the weighted homogeneous version of the unit sliding mode control (Dorling \& Zinober, 1986; Utkin et al., 2009).

Note that in presented control scheme instead of homogeneous norm (3) another homogeneous function of degree 1 can be used. In this case the main difference is in calculating the parameter $\alpha$. Also if we choose instead of homogeneous norm implicitly defined homogeneous function equal to $V$, then for $m=l=1$ we obtain implicitly defined Lyapunov function

$$
Q=x^{T} D_{r}\left(V^{-1}\right) P D_{r}\left(V^{-1}\right) x-1
$$


and control $u=V^{1-\mu} k D_{r}\left(V^{-1}\right) x, V \in \mathbb{R}_{+}: Q(V, x)=0$ as in (Polyakov et al., 2015; Polyakov, Efimov, \& Perruquetti, 2016).

Convergence within a fixed interval of time irrespectively of its initial conditions can be achieved by changing the degree of homogeneity in hybrid control algorithm. The following discontinuous fixed-time controller is based on time dependent switching scheme for the first time presented in (Angulo, Moreno, \& Fridman, 2013) for an observer.

In this case for $m_{1}, m_{2} \in \mathbb{R}_{+}: m_{1}=m_{2}+2 \mu, m_{1}>n \mu, l>(n-1) \mu, \mu \in(0,1]$ denote vectors of weights $r_{1}=(l+(n-1) \mu, l+(n-2) \mu, \cdots, l)^{T}, r_{2}=(l-(n-1) \mu, l-(n-$ 2) $\mu, \cdots, l)^{T}$, homogeneous norms $\|x\|_{r_{1}},\|x\|_{r_{2}}$ and matrices $D_{1}(\lambda)=\operatorname{diag}\left\{\lambda^{r_{1}(i)}\right\}_{i=1}^{n}$, $\tilde{H}_{1}=\operatorname{diag}\left\{-m_{1}+(n+1-i) \mu\right\}_{i=1}^{n}, D_{2}(\lambda)=\operatorname{diag}\left\{\lambda^{r_{2}(i)}\right\}_{i=1}^{n}, \tilde{H}_{2}=\operatorname{diag}\left\{-m_{2}-(n+\right.$ $1-i) \mu\}_{i=1}^{n}, H_{1}(\lambda)=\operatorname{diag}\left\{\lambda^{-m_{1}+(n+1-i) \mu}\right\}_{i=1}^{n}, H_{2}(\lambda)=\operatorname{diag}\left\{\lambda^{-m_{2}-(n+1-i) \mu}\right\}_{i=1}^{n}$.

Define homogeneous functions of degree 1 :

$$
\begin{aligned}
& V_{1}(x)=\left(x^{T} H_{1}\left(\frac{1}{\|x\|_{r_{1}}}\right) P H_{1}\left(\frac{1}{\|x\|_{r_{1}}}\right) x\right)^{\frac{1}{2\left(m_{1}+l-\mu\right)}}, \\
& V_{2}(x)=\left(x^{T} H_{2}\left(\frac{1}{\|x\|_{r_{2}}}\right) P H_{2}\left(\frac{1}{\|x\|_{r_{2}}}\right) x\right)^{\frac{1}{2\left(m_{2}+l+\mu\right)}} .
\end{aligned}
$$

For $V_{1}$ and $V_{2}$ according to (5) define $c_{1_{1}}, c_{2_{1}}$ and $c_{1_{2}}, c_{2_{2}}$ correspondingly.

Theorem 4.2. If the system of matrix inequalities (6),

$$
\begin{gathered}
\alpha_{1}\left(\tilde{H}_{1} X+X \tilde{H}_{1}\right)+A X+X A^{T}+b y+y^{T} b^{T} \leq-\gamma_{1} X, \\
-\alpha_{1}\left(\tilde{H}_{1} X+X \tilde{H}_{1}\right)+A X+X A^{T}+b y+y^{T} b^{T} \leq-\gamma_{1} X, \\
\alpha_{2}\left(\tilde{H}_{2} X+X \tilde{H}_{2}\right)+A X+X A^{T}+b y+y^{T} b^{T} \leq-\gamma_{2} X, \\
-\alpha_{2}\left(\tilde{H}_{2} X+X \tilde{H}_{2}\right)+A X+X A^{T}+b y+y^{T} b^{T} \leq-\gamma_{2} X,
\end{gathered}
$$

is feasible for some $X \in \mathbb{R}^{n \times n}, y \in \mathbb{R}^{1 \times n}, \gamma_{1}, \gamma_{2} \in \mathbb{R}_{+}$, where $\alpha_{1}$, $\alpha_{2}$ are calculated according to (9) with $r_{1}$ and $r_{2}$ correspondingly, $P=X^{-1}, k=y X^{-1}$, then the control of the form

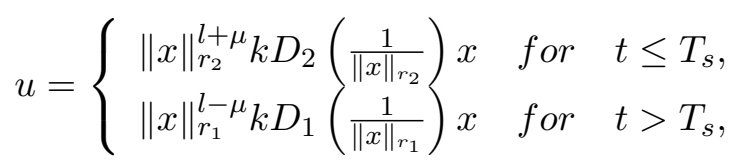

for some $T_{s} \in \mathbb{R}_{+}$stabilizes the origin of the system (1) in a fixed time

$$
T\left(x_{0}\right) \leq 2\left(m_{1}+l-\mu\right) \mu^{-1}\left(\frac{c_{2_{2}}^{\mu}}{\gamma_{2} E^{\mu}}+\frac{B^{\mu}}{\gamma_{1} c_{1_{1}}^{\mu}}\right)
$$


where $E=\left(\frac{2\left(m_{1}+l-\mu\right) c_{2_{2}}^{\mu}}{\mu \gamma_{2} T_{s}}\right)^{\frac{1}{\mu}}$,

$$
B=\max _{V_{2}=E} V_{1}
$$

Proof. Similarly to the proof of Theorem 4.1 it can be shown that if the inequalities (6), (12)-(15) hold, then

$$
\dot{V}_{2} \leq-\frac{\gamma_{2} V_{2}^{1+\mu}}{2\left(m_{2}+l+\mu\right) c_{2_{2}}^{\mu}}
$$

and

$$
\dot{V}_{1} \leq-\frac{\gamma_{1} c_{1_{1}}^{\mu} V_{1}^{1-\mu}}{2\left(m_{1}+l-\mu\right)}
$$

Consider the following sets

$$
\mathbb{B}_{1}=\left\{x \in \mathbb{R}^{n}: V_{1} \leq B\right\}
$$

and

$$
\mathbb{B}_{2}=\left\{x \in \mathbb{R}^{n}: V_{2} \leq E\right\} .
$$

By (18), it is obvious, that $\mathbb{B}_{2} \subset \mathbb{B}_{1}$. According to Theorem 3.5 and (19) the set $\mathbb{B}_{2}$ is fixed-time attractive with $T_{\max }=T_{s}=\frac{2\left(m_{2}+l+\mu\right) c_{2_{2}}^{\mu}}{\mu \gamma_{2} E^{\mu}}=\frac{2\left(m_{1}+l-\mu\right) c_{2_{2}}^{\mu}}{\mu \gamma_{2} E^{\mu}}$. When $x$ reaches the set $\mathbb{B}_{2} \subset \mathbb{B}_{1}$, for $t>T_{s}$ the system will become finite-time stable and it will converge to the origin in a time, bounded by $T_{1}=\frac{2\left(m_{1}+l-\mu\right) B^{\mu}}{\mu \gamma_{1} c_{1}^{\mu}}$. Then the control (16) stabilizes the origin of the system (1) in a fixed time $T\left(x_{0}\right) \leq T_{1}+T_{s}$, that is equal to $(17)$.

Note, that the parameter $T_{s} \in \mathbb{R}_{+}$can be arbitrarily chosen.

Similarly to finite-time case, remarks analogous to $2-6$ can be presented for fixedtime control algorithm. For example, the fixed-time controller is constructed by switching homogeneity properties of the system: it is homogeneous with positive degree $\mu$ for $t \leq T_{s}$ and with negative degree $-\mu$ for $t>T_{s}$.

Remark 9. Analogously to Remark 7, due to the fact that the closed-loop system (1), (16) is homogeneous with negative degree $-\mu$ for $t \geq T_{s}$ it is globally asymptotically stable with respect to a compact set containing the origin in presence of any delay.

The convergence rate of proposed control algorithms can be accelerated via timerescaling in similar way, as in (Efimov, Levant, Polyakov, \& Perruquetti, 2016; Harmouche et al., 2016; Levant \& Dvir, 2014).

Remark 10. For $\lambda>1$ and $N=\operatorname{diag}\left\{\lambda^{1-i}\right\}_{i=1}^{n}$ rewrite the control (16) (or control (10)) as $\bar{u}(x)=\lambda^{n} u(N x)$. Then for $\bar{x}=N x, \bar{t}=\lambda t, \bar{T}_{s}=T_{s} / \lambda$ we obtain $\dot{\bar{x}}=A \bar{x}(\bar{t})+b u(\bar{x}(\bar{t}))$ and the following estimates of settling-time function: $T\left(x_{0}\right) \leq \frac{T_{1}+T_{s}}{\lambda}$ for the control (16) and $T\left(x_{0}\right) \leq \frac{2(m+l-\mu) V\left(\bar{x}_{0}\right)^{\mu}}{\mu \gamma c_{1}^{\mu} \lambda}$ for the control (10). 
The result of Theorem 4.2 can be easily transformed for the case of continuous control law as follows.

Theorem 4.3. If the system of matrix inequalities (6), (12)-(15),

$$
A X+X A^{T}+b y+y^{T} b^{T} \leq-\tilde{\alpha} X,
$$

is feasible for some $X \in \mathbb{R}^{n \times n}, y \in \mathbb{R}^{1 \times n}, \gamma_{1}, \gamma_{2}, \tilde{\alpha} \in \mathbb{R}_{+}$, where $\alpha_{1}$, $\alpha_{2}$ are calculated according to (9) with $r_{1}$ and $r_{2}$ correspondingly, $P=X^{-1}, k=y X^{-1}$, then the control of the form

$$
u=\left\{\begin{array}{l}
\|x\|_{r_{2}}^{l+\mu} k D_{2}\left(\frac{1}{\|x\|_{r_{2}}}\right) x \text { for } \quad\|x\|_{r_{2}} \geq 1, \\
k x \quad \text { for }\|x\|_{r_{1}} \geq 1 \text { and }\|x\|_{r_{2}}<1, \\
\|x\|_{r_{1}}^{l-\mu} k D_{1}\left(\frac{1}{\|x\|_{r_{1}}}\right) x \text { for }\|x\|_{r_{1}}<1,
\end{array}\right.
$$

stabilizes the origin of the system (1) in a fixed time

$$
T\left(x_{0}\right) \leq 2\left(m_{1}+l-\mu\right)\left(\frac{c_{2_{2}}^{\mu}}{\mu \gamma_{2} c_{1_{2}}^{\mu}}+\frac{c_{2_{1}}^{\mu}}{\mu \gamma_{1} c_{1_{1}}^{\mu}}+\frac{\ln \left(c_{2_{2}} / c_{1_{1}}\right)}{\tilde{\alpha}}\right) .
$$

Proof. Consider the following sets

$$
\mathbb{C}_{1}=\left\{x \in \mathbb{R}^{n}:\|x\|_{r_{1}} \leq 1\right\}
$$

and

$$
\mathbb{C}_{2}=\left\{x \in \mathbb{R}^{n}:\|x\|_{r_{2}} \leq 1\right\}
$$

Since $r_{1}(i) \geq r_{2}(i)$ for $i=\overline{1, n}$ we have $\mathbb{C}_{1} \subseteq \mathbb{C}_{2}$. According to Theorem 3.5 and Theorem 4.2 the set $\mathbb{C}_{2}$ is fixed-time attractive with $T_{2}=T_{\max }=\frac{2\left(m_{1}+l-\mu\right) c_{2_{2}}^{\mu}}{\mu \gamma_{2_{1}}^{\mu}}$. When $x$ reaches the set $\mathbb{C}_{2}$, the system will become exponentially stable with $u=k x, V=$ $\left(x^{T} P x\right)^{\frac{1}{2\left(m_{1}+l-\mu\right)}}$ and due to $(20)$ will reach the set $\mathbb{C}_{1}$ in a time, bounded by $T_{\text {lin }}=$ $2\left(m_{1}+l-\mu\right) \frac{\ln \left(c_{2_{2}} / c_{1_{1}}\right)}{\tilde{\alpha}}$. Finally, inside the set $\mathbb{C}_{1}$ the closed-loop system is finitetime stable with $T_{1}=\frac{2\left(m_{1}+l-\mu\right) c_{2_{1}}^{\mu}}{\mu \gamma_{1} c_{11}^{\mu}}$. Then the control (21) stabilizes the origin of the system (1) in a fixed time $T\left(x_{0}\right) \leq T_{1}+T_{2}+T_{\text {lin }}$, that is equal to (22).

Remark 11. In comparison with the control law (16), since the closed-loop system (1), (21) is $r$-homogeneous with positive degree $\mu$ on the set $\left\{x \in \mathbb{R}^{n}:\|x\|_{r_{2}} \geq 1\right\}$, then it preserves robustness with respect to some delay $\tau \leq \tau_{0}, \tau_{0} \in \mathbb{R}_{+}$(according to Efimov, Polyakov, et al., 2016; Zimenko et al., 2017).

Remark 12. Presented results can be easily extended to linear MIMO systems using block decomposition like in (Polyakov et al., 2016). Thereby, feedback linearizable nonlinear systems $\dot{x}=f(x)+G(x) u$ can also be stabilized by presented control laws (see, for example, Isidori, 1995). 


\section{Simulation results}

Let us demonstrate the performance of presented control laws using the system of triple integrator. Using Theorem 4.1 the finite-time stabilizing controller is designed for this system in the form (10) with the parameters $l=45, m=1.21, \mu=0.4$, $\rho=91.6$, where the matrix $P \in \mathbb{R}^{3 \times 3}$ and the vector $k \in \mathbb{R}^{1 \times 3}$ are obtained from the inequalities (6)-(8):

$$
\begin{gathered}
P=\left(\begin{array}{lll}
1.2382 & 0.4564 & 0.0371 \\
0.4564 & 0.2068 & 0.0202 \\
0.0371 & 0.0202 & 0.0035
\end{array}\right), \\
k=\left(\begin{array}{lll}
-122.7548 & -63.2132 & -9.4683
\end{array}\right) .
\end{gathered}
$$

Obtained estimation of the settling-time function for $x_{0}=\left(\begin{array}{lll}0 & 0 & 1\end{array}\right)^{T}$ is $T\left(x_{0}\right) \leq$ 712.1066 .

The parameters of the fixed-time stabilizing controller (16) were selected solving the LMIs (6), (12)-(15) with the parameters $l=45, m_{1}=1.21, m_{2}=0.41, \mu=0.4$, $\rho_{1}=\rho_{2}=91.6$ :

$$
\begin{gathered}
P=\left(\begin{array}{ccc}
24.1416 & 16.8444 & 3.0117 \\
16.8444 & 14.9767 & 3.1131 \\
3.0117 & 3.1131 & 1.0985
\end{array}\right), \\
k=\left(\begin{array}{lll}
-19.9578 & -20.9022 & -6.7877
\end{array}\right) .
\end{gathered}
$$

The parameter $T_{s}$ has been chosen equal to 2 . Obtained estimation of the settling-time function is $T\left(x_{0}\right) \leq 921.1276$. The simulation has been carried out for $x_{0}=\left(\begin{array}{lll}0 & 0 & 10\end{array}\right)^{T}$.

The parameters of the fixed-time stabilizing controller (21) were selected solving the LMIs (6), (12)-(15), (20) with the same parameters as in previous example and $\tilde{\alpha}=2.6966, T\left(x_{0}\right) \leq 922.84$. The simulation has been carried out for $x_{0}=\left(\begin{array}{lll}0 & 0 & 10\end{array}\right)^{T}$.

Simulations for finite-time and fixed-time convergence accelerated with $\lambda=3$ and the same initial conditions have also been carried out for the control laws (10) and (16) with $T\left(x_{0}\right) \leq 232.7987$ and $T\left(x_{0}\right) \leq 307.0425$, correspondingly. All simulations results are presented in Fig.1-8.

Note, that presented estimations of the settling-time function are quite conservative due to conservative estimation of $\alpha_{\max }$ in (9) and, thus, of the parameter $\gamma$. For example, during the simulation presented in Fig.1, the parameter $\alpha(x)$ did not exceed the value 1.257 and using this value as $\alpha_{\max }$ the estimation of the settling-time function $T\left(x_{0}\right) \leq 67.65$ could be obtained. Thus, the search for another $r$-homogeneous function of degree 1 instead of (3) and/or less conservative estimate of the parameter $\alpha_{\max }$ can be considered as the subject for a future research.

\section{Conclusions}

The paper is focused on finite-time and fixed-time control for integrator chains of arbitrary order. Obtained finite-time control design method consist in appropriate 


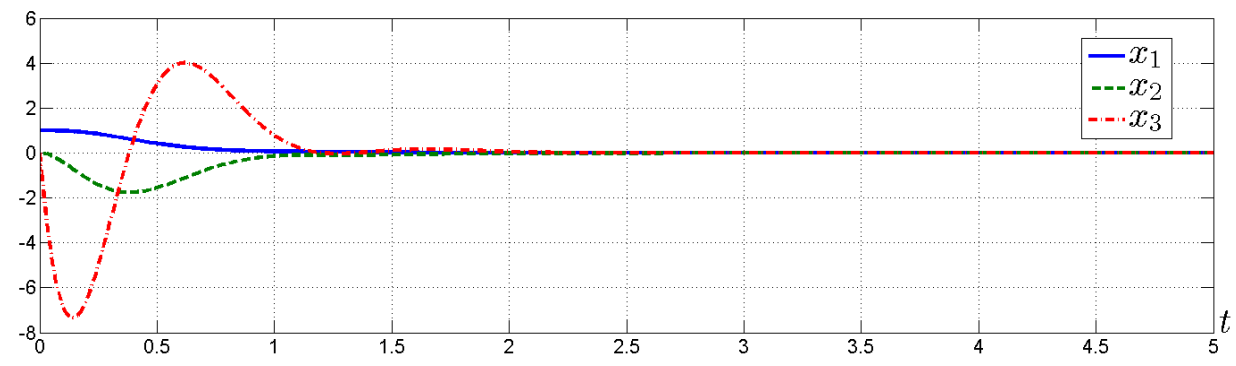

Figure 1. System states versus time for finite-time control

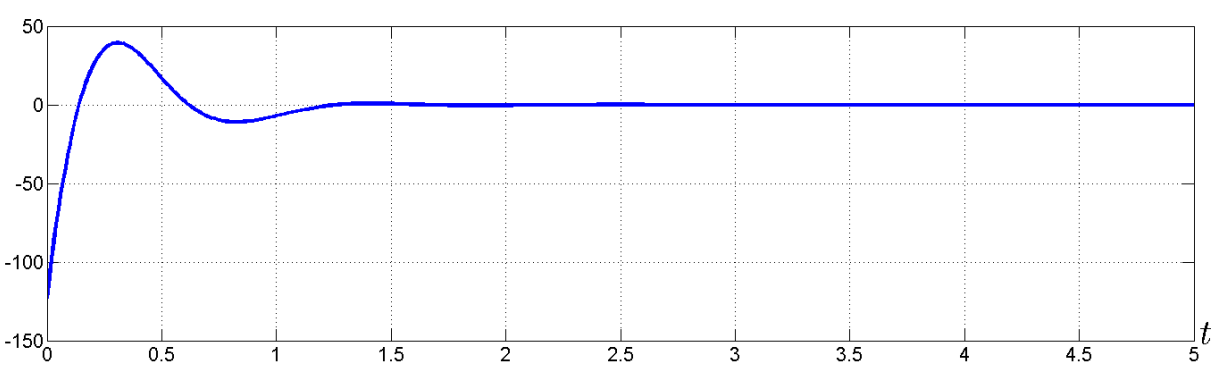

Figure 2. Control law (10) versus time

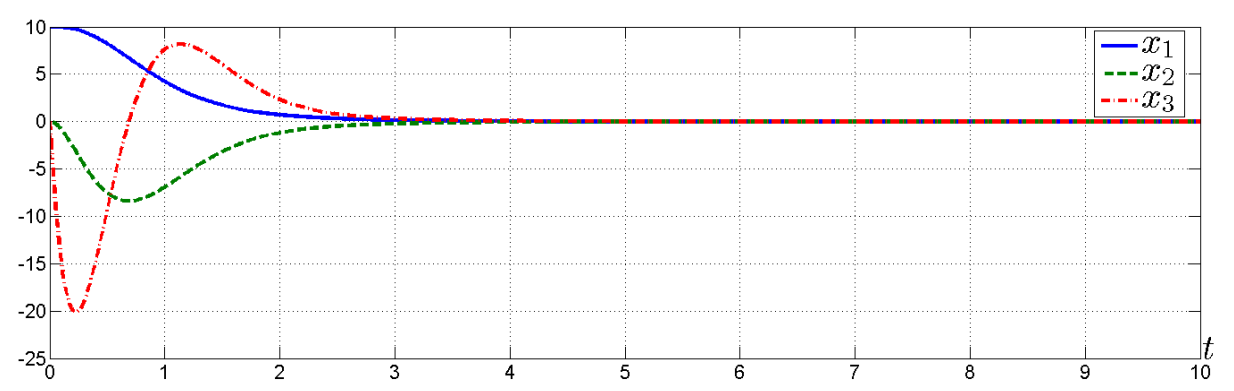

Figure 3. System states versus time for fixed-time control (16)

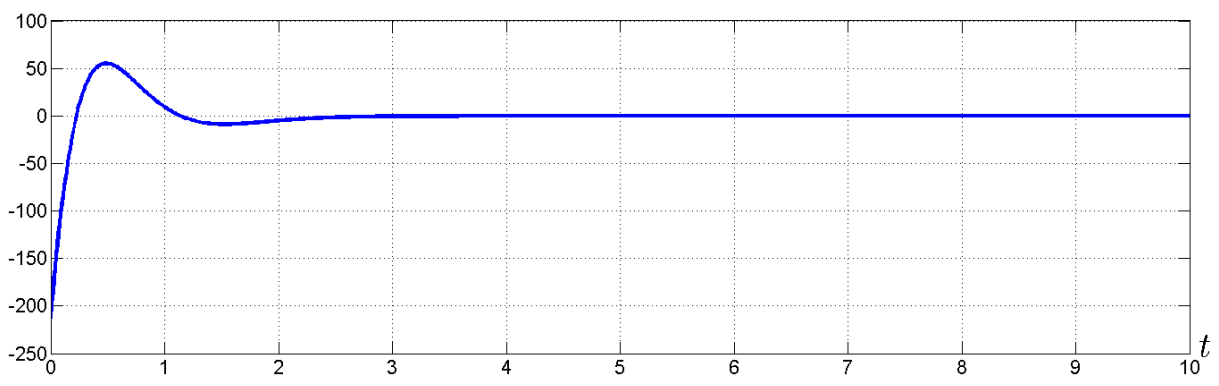

Figure 4. Control law (16) versus time 


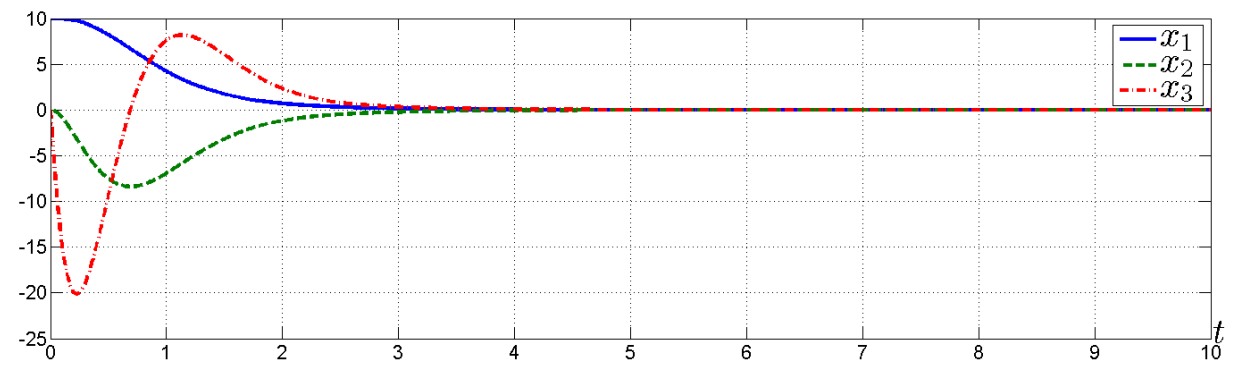

Figure 5. System states versus time for fixed-time control (21)

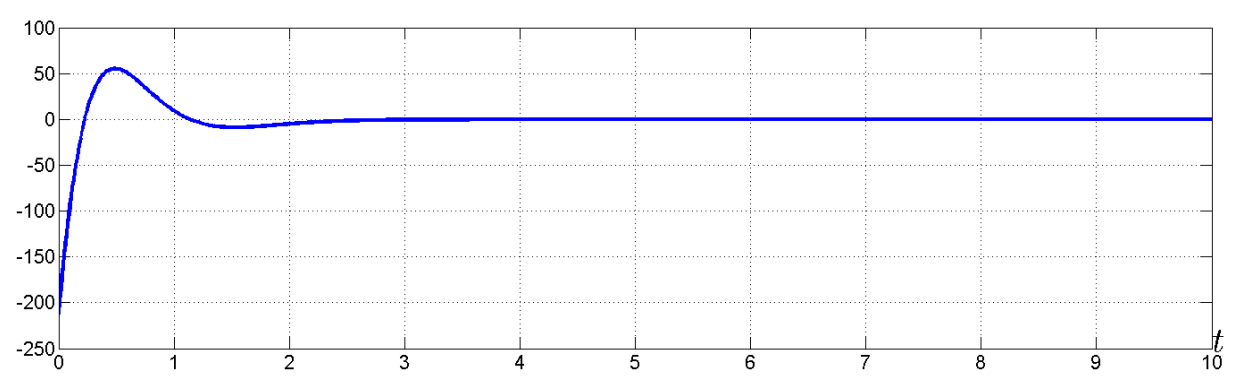

Figure 6. Control law (21) versus time

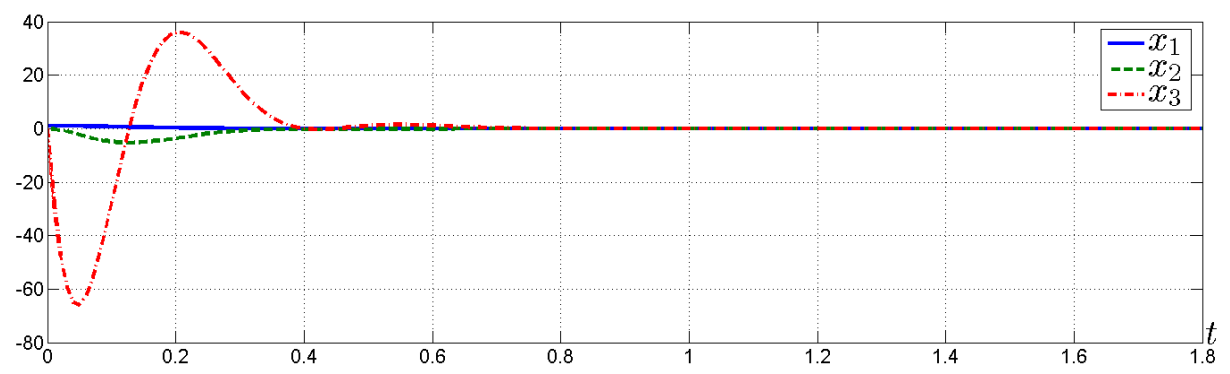

Figure 7. System states versus time for accelerated finite-time control (10)

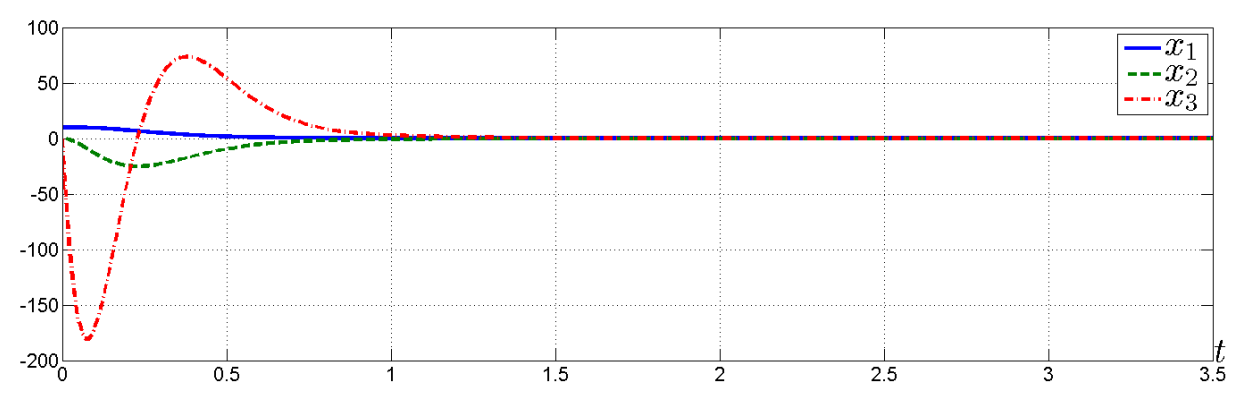

Figure 8. System states versus time for accelerated fixed-time control (16) 
modification of control law presented in (Zimenko et al., 2016) and use of analysis based on explicitly defined Lyapunov function. Based on this result simple procedure of parameters tuning and new estimates for settling-time function were obtained. The fixed-time control algorithms for chain of integrators are presented. It is shown that fixed-time convergence can be achieved by changing the homogeneity degree in hybrid control algorithms. The performance of obtained control algorithms is demonstrated through simulations.

A detailed study of the presented control algorithms on robustness analysis with respect to disturbances, uncertainties, delays and extension of these results on wider class of systems goes beyond the scope of the paper providing the subject for a future research.

\section{Funding}

This work is supported by the Russian Science Foundation under grant 17-19-01422.

\section{References}

Andrieu, V., Praly, L., \& Astolfi, A. (2008). Homogeneous approximation, recursive observer and output feedback. SIAM Journal of Control and Optimization, 47(4), 1814-1850.

Angulo, M. T., Moreno, J., Fridman, L. (2013). Robust exact uniformly convergent arbitrary order differentiator. Automatica, 49, 2489-2495.

Bacciotti, A., \& Rosier, L. (2005). Lyapunov Functions and Stability in Control Theory. Springer.

Bernuau, E., Polyakov, A., Efimov, D., \& Perruquetti, W. (2013). Verification of ISS, iISS and IOSS properties applying weighted homogeneity. System and Control Letters, 62(12), 1159-1167.

Bhat, S., \& Bernstein D. (2000). Finite-time stability of continuous autonomous systems. SIAM Journal of Control and Optimization, 38(3), 751-766.

Chernousko, F. L., Ananevskii, I. M., \& Reshmin, S. A. (2008). Control of nonlinear dynamical systems: methods and applications. Berlin: Springer-Verlag.

Cruz-Zavala, E., \& Moreno, J. (2017). Homogeneous High Order Sliding Mode design: A Lyapunov approach. Automatica, 80, 232-238.

Dorling, C. M., Zinober, A. S. I. (1986). Two approaches to hyperplane design in multivariable variable structure control systems. International Journal of Control, 44(1), 65-82.

Efimov, D., Levant, A., Polyakov, A., \& Perruquetti W. (2016). Supervisory Acceleration of Convergence for Homogeneous Systems. International Journal of Control, 1-11.

Efimov, D., Polyakov, A., Perruquetti, W., \& Richard, J.-P. (2016). Weighted homogeneity for time-delay systems: Finite-time and independent of delay stability. IEEE Trans. Automatic Control, 61(1), 210-215.

Filippov, A. (1988). Differential equations with discontinuous right-hand sides. Dordrecht, Kluwer.

Harmouche, M., Laghrouche, S., Chitour, Y., \& Hamerlain, M. (2016). Stabilisation of perturbed chains of integrators using Lyapunov-based homogeneous controllers. International Journal of Control, 2631-2640.

Isidori, A. (1995). Nonlinear Control Systems. Springer-Verlag, N. Y. Inc.

Levant, A., \& Dvir, Y. (2014). Accelerated high-order MIMO sliding mode control. Proc. of the 13th International Workshop on Variable Structure Systems, 1-6.

Orlov, Y. (2004). Finite Time Stability and Robust Control Synthesis of Uncertain Switched Systems. SIAM Journal of Control and Optimization, 43(4), 1253-1271. 
Orlov, Y., Aoustin, Y., \& Chevallereau, C. (2011). Finite Time Stabilization of a Perturbed Double Integrator-Part I: Continuous Sliding Mode-Based Output Feedback Synthesis. IEEE Transactions on Automatic Control, 56(3), 614-618.

Polyakov, A. (2012). Nonlinear feedback design for fixed-time stabilization of linear control systems. IEEE Transactions on Automatic Control, 57(8), 2106-2110.

Polyakov, A., Efimov, D., \& Perruquetti, W. (2015). Finite-time and fixed-time stabilization: Implicit Lyapunov function approach. Automatica, 51, 332-340.

Polyakov, A., Efimov, D., \& Perruquetti, W. (2016). Robust Stabilization of MIMO Systems in Finite/Fixed Time. Int. J. Robust. Nonlinear Control, 26, 69-90.

Sanchez, T., \& Moreno, J. (2013). On a sign controller for the triple integrator. Proc. of the 52nd Annual Conference on Decision and Control (CDC), 3566-3571.

Sánchez-Torres, J. D., Sanchez, E. N., \& Loukianov, A. G. (2015). Predefined-time stability of dynamical systems with sliding modes. Proceedings of American Control Conference (ACC), $5842-5846,2015$.

Sua, Y., \& Zheng, C. (2015). Robust finite-time output feedback control of perturbed double integrator. Automatica, 60, 86-91.

Trivedi, P., \& Bandyopadhyay, B. (2011). Finite-time stabilization of uncertain triple integrator with only switch and gain. Proc. of the 37th Annual Conference on IEEE Industrial Electronics Society (IECON 2011), 3942-3946.

Utkin, V., Guldner, J., \& Shi, J. (2009). Sliding Mode Control in Electro-Mechanical Systems. CRC Press.

Zimenko, K., Efimov, D., Polyakov, A., \& Perruquetti, W. (2017). A note on delay robustness for homogeneous systems with negative degree. Automatica, 79, 178-184.

Zimenko, K., Polyakov, A., \& Efimov D. (2016). Stabilization of Chain of Integrators with Arbitrary Order in Finite-time. Proceedings of the 54th IEEE Conference on Decision and Control, 4637-4641.

Zimenko, K., Polyakov, A., Efimov, D., \& Perruquetti, W. (in press). Finite-time and fixedtime stabilization for integrator chain of arbitrary order. European Control Conference (ECC 2018).

Zubov, V. (1958). On systems of ordinary differential equations with generalized homogeneous right-hand sides. (in Russian). Izvestia vuzov. Mathematica, 1, 80-88. 